\title{
Etude du transfert de matière lors de l'ultrafiltration de lactosérum doux sur membrane minérale
}

\author{
Christine TADDEI*, P. AIMAR**, G. DAUFIN* et V. SANCHEZ** \\ * I.N.R.A., Laboratoire de Recherches de Technologie laitière \\ 65 , rue de Saint-Brieuc, 35042 Rennes cedex, France \\ ** Laboratoire de Chimie Physique et Electrochimie, U.A., C.N.R.S. 192 \\ 118 , route de Narbonne, 31062 Toulouse cedex, France
}

\section{Résumé}

On étudie le transfert de matière lors de l'ultrafiltration de lactosérum doux sur membrane minérale (Carbosep, type M4), à l'aide d'un tube, motif élémentaire d'une installation industrielle.

Les paramètres opératoires sont la vitesse tangentielle $\left(1,8\right.$ à $\left.4,0 \mathrm{~m} . \mathrm{s}^{-1}\right)$, la différence de pression transmembranaire moyenne $\left(0,3\right.$ à $\left.5,7 \cdot 10^{5} \mathrm{~Pa}\right)$, la température $(25$ ou $\left.50^{\circ} \mathrm{C}\right)$ et le temps $(2 \min$ à $8 \mathrm{~h}$ ).

Dans le cas des solutés ioniques et non protéiques du lactosérum, la loi de Darcy s'applique et la viscosité dynamique de la solution explique la baisse de perméabilité par rapport à l'eau. Ces solutés ne sont pas retenus par la membrane et ne provoquent pas de colmatage grave de celle-ci, à l'exception du phosphate de calcium.

Lors de l'ultrafiltration du lactosérum, les vitesses de perméation et les taux de rétention en protéines solubles diminuent pendant la première heure, vraisemblablement en raison de phénomènes d'adsorption et de polarisation. Ensuite, le flux d'ultrafiltration diminue moins rapidement et les taux de rétention augmentent, en raison de la formation d'un dépôt superficiel.

Les paramètres opératoires (vitesse tangentielle, différence de pression transmembranaire et durée) ont une incidence variable sur les trois grandeurs caractéristiques du transfert de matière : densité de flux, rétention en protéines, niveau de colmatage de la membrane. L'analyse de leurs influences respectives permet de proposer des lignes directrices pour la conduite d'une opération d'ultrafiltration, en fonction des performances recherchées. Elle permet, en outre, de mieux cerner les mécanismes possibles des phénomènes limitant le transfert de matière.

Mots clés: Transfert de matière - Lactosérum doux - Ultrafiltration - Membranes minérales.

\section{Summary}

\section{Mass transfer during sweet whey ultrafiltration on mineral membrane}

Mass transfer has been studied during sweet whey ultrafiltration on mineral membrane (Carbosep M4 Type) with a monotube. 
Operating parameters are : tangential flow velocity $\left(1.8\right.$ to $\left.4.0 \mathrm{~m} . \mathrm{s}^{-1}\right)$; mean pressure between the inlet and the outlet of the tubular membrane $\left(0.3\right.$ to $\left.5.710^{5} \mathrm{~Pa}\right)$; temperature $\left(25\right.$ or $\left.50^{\circ} \mathrm{C}\right)$; time $(2 \mathrm{~min}$ to $8 \mathrm{~h})$.

In the case of solutions containing ionic and non proteinic solutes of sweet whey, Darcy's law may be applied and dynamic viscosity of such solutions explains the permeability decrease towards pure water. These components are not retained by the membrane and do not lead to a severe fouling, except the case of calcium phosphate. Nevertheless, presence of lactose lowers fluxes, probably in relation with an adsorption phenomenon.

During sweet whey ultrafiltration, small components unlike proteins are not retained by the membrane whatever the operating parameters. During a first step, fluxes and protein retention lower, probably as a result of adsorption and polarization phenomena. During a second step flux decrease becomes slower and protein retention increases, related to an accumulation of matter.

Operating parameters (tangential flow velocity, pressure and time) have a great effect on mass transfer characteristics : fluxes, protein retention and membrane fouling level. The latter is evaluated by the measure of membrane hydraulic resistance before and after each run. Increasing flow velocity at a constant pressure leads to improve fluxes but proteins retention decreases and fouling becomes less important. On the other hand, increasing pressure at a constant flow velocity generally enhances fluxes, retention but fouling as well. Of course, time plays an important part on membrane performances as described before. Therefore we propose some suggestions on driving an ultrafiltration operation. tations.

Analyzing all these results allows a better understanding of mass transfer limi-

Key words: Mass transfer - Ultrafiltration - Sweet whey - Mineral membrane.

\section{Introduction}

L'ultrafiltration est largement utilisée comme procédé de concentration des protéines du lactosérum. Pour une qualité donnée de produit final, les performances d'un équipement sont définies par divers critères techniques et économiques : flux d'ultrafiltration, rétention des protéines, fréquence des nettoyages, durée de vie des membranes...

Une amélioration sensible des débits de perméation a été obtenue par le prétraitement du lactosérum (HicKey et al., 1980; AMUNDSON et al., 1982; MauboIs, 1982). Ces traitements de nature physico-chimique ont permis de réduire le colmatage de surface et intramembranaire et donc d'accroître les flux.

Mais il n'en reste pàs moins vrai que les phénomènes limitant le transfert de l'ensemble solutés-solvant au travers d'une membrane d'ultrafiltration sont mal connus et que leur élucidation, conduisant à leur maîtrise, permettrait d'optimiser les performances d'une installation dans un contexte technologique donné (composition et caractéristiques du lactosérum à traiter, pouvoir de coupure et nature des membranes mises en œuvre, caractéristiques et composition du rétentat souhaité). 
Diverses tentatives ont été faites pour améliorer nos connaissances sur le transfert de matière en ultrafiltration. LAFAILle (1984) et Clifton et al. (1984), utilisant des solutions de molécules synthétiques (polyéthylène-glycol, dextrane), ont précisé l'effet de deux phénomènes limitants : la formation d'une couche de polarisation et le développement d'une pression osmotique antagoniste à la pression appliquée. Le cas de fluides biologiques a été approché par FANE et al. (1983) et BAKLOUTI et al. (1984) qui ont mis en contact des solutions de sérum albumine bovine et des membranes polymères. Ils ont ainsi pu préciser respectivement la contribution de la membrane, de l'adsorption de protéines et de la couche de polarisation à l'accroissement de la résistance au transfert de matière. Mais la transposition de ces résultats au traitement du lactosérum n'est pas réalisable en raison de la complexité du produit. De plus, il est certain que la nature des composants constitutifs de la membrane intervient dans ces phénomènes limitants et que c'est le couple membrane-solution traité qui doit être pris en considération compte tenu des interactions membranes/solutés susceptibles d'intervenir (MatThiasson, 1983). En outre, les résultats obtenus sur membranes polymères ne sont pas toujours transposables aux membranes minérales (GouRdoN, 1980) et vice versa.

Sur un autre plan, peu d'études ont été consacrées à l'évolution des flux (Kuo et Cheryan, 1983) et des taux de rétention protéique en fonction du temps. Or il s'agit pourtant de données essentielles pour évaluer la rentabilité technico-économique d'une installation d'ultrafiltration du lactosérum.

En conséquence, l'objectif de ce travail était de contribuer à améliorer les connaissances sur les phénomènes limitant le transfert de matière lors de l'ultrafiltration de lactosérum doux sur membrane minérale. Ainsi, avons-nous étudié le transport transmembranaire des composants du lactosérum doux en fonction des paramètres opératoires (vitesse tangentielle, différence de pression transmembranaire moyenne) et de la durée du traitement. Dans un premier temps, une méthodologie expérimentale a été définie dans le but de permettre la comparaison des résultats. Puis le transfert a été étudié d'abord à l'aide de solutions modèles simulant progressivement la composition du lactosérum (solutés ioniques et non protéiques), puis avec le lactosérum lui-même.

\section{Matériel et méthodes}

\section{A. Dispositif expérimental}

Le banc d'ultrafiltration comportait une pompe de recirculation (pompe volumétrique à vis excentrée : $120-12001 . \mathrm{h}^{-1}$ ), un débitmètre à flotteur (précision $\pm 1 \%$ ) et un tube d'ultrafiltration de longueur $1,20 \mathrm{~m}$, de diamètre intérieur $6.10^{-3} \mathrm{~m}$ et de surface membranaire $2,26 \cdot 10^{-2} \mathrm{~m}^{2}$ (Carbosep, type M4 seuil de coupure : 40000 Dalton : support carbone microporeux et couche filtrante en oxyde de zirconium). Deux manomètres piézoélectriques (précision $\pm 1 \%$ ) permettaient de connaître la perte de charge et la différence de pression transmembranaire, réglée au moyen d'une vanne à pointeau placée à la sortie du tube. 
La température du fluide était régulée à $\pm 0,1^{\circ} \mathrm{C}$ au moyen d'un serpentin d'eau chaude immergé dans le bac de recirculation.

Les valeurs des paramètres opératoires étaient :

- vitesse tangentielle moyenne de circulation du fluide (V) : $1,8(\mathrm{Re}=$ $12000) ; 3,0 ; 3,6$ et $4,0 \mathrm{~m} . \mathrm{s}^{-1}$;

- pression transmembranaire $(\Delta \mathrm{P})$ : moyenne arithmétique entre la pression d'entrée et de sortie : 0,$3 ; 1,0 ; 2,0 ; 4,0 ; 5,0 ; 5,7\left(\times 10^{5} \mathrm{~Pa}\right)$;

- température $(\theta): 25$ et $50^{\circ} \mathrm{C}$;

- temps (t) : 30 min à $8 \mathrm{~h}$.

\section{B. Caractérisation du transfert de matière}

\section{Densité de flux $(J)$}

Le débit de perméat était mesuré à $2 \%$ près au moyen d'une balance à tare automatique. La densité du flux était exprimée en $1 . \mathrm{h}^{-1} \cdot \mathrm{m}^{-2}$.

\section{Résistance hydraulique de la membrane (Rh)}

Par application de la loi de Darcy, le flux de perméat dans le cas d'un solvant pur et pour des pressions inférieures à $10 \times 10^{5} \mathrm{~Pa}$ s'exprime ainsi :

$$
\mathbf{J}=\mathbf{L p} . \Delta \mathrm{P}
$$

avec Lp : perméabilité de la membrane. Si l'on pose $\mathrm{L}_{\mathrm{p}}=1 / \eta_{\text {eau }} \times \mathrm{Rh}$, dans le cas de transfert d'eau pure au travers d'une membrane la relation (1) devient :

$\mathrm{J}_{\text {ean }}=$ densité de flux $\left(\mathrm{m}^{3} \cdot \mathrm{s}^{-1} \cdot \mathrm{m}^{-2}\right)$

$$
\mathrm{J}_{\text {eau }}=\frac{\Delta \mathrm{P}}{\eta_{\text {eau }} \times \mathrm{Rh}}
$$

avec $\mathrm{Rh}=$ résistance hydraulique de la membrane $\left(\mathrm{m}^{-1}\right)$ et $\eta_{\text {eau }}=$ viscosité dynamique de l'eau (Pa.s) à la température considérée.

Les mesures de résistances hydrauliques effectuées sur la membrane propre dans des conditions opératoires données $\left(1,8 \mathrm{~m} \cdot \mathrm{s}^{-1} ; 2,80 \times 10^{5} \mathrm{~Pa} ; 25^{\circ} \mathrm{C}\right)$ ont mis en évidence une baisse de perméabilité de la membrane en $8 \mathrm{~h}$ allant de $3 \%$ dans le cas de la filtration d'eau fraîchement distillée à $30 \%$ et plus dans le cas de la filtration d'eau du réseau ou d'eau distillée datant de plusieurs jours.

Par conséquent seule l'eau fraîchement distillée et de conductivité inférieure à $4 \mu \mathrm{S} \cdot \mathrm{m}^{-1}(\mathrm{pH}=6,0)$ était utilisée pour mesurer le flux à partir duquel on a calculé la résistance hydraulique (éq. 2).

Toutes les mesures étaient réalisées à $1,8 \mathrm{~m} \cdot \mathrm{s}^{-1}$ sous une différence de pression transmembranaire de $1,00.10^{5} \mathrm{~Pa}$.

La mesure de la résistance hydraulique de la membrane avant l'essai d'ultrafiltration permet de s'assurer que celle-ci est toujours dans le même état de propreté. La mesure de la résistance hydraulique finale caractérise l'état de la membrane vis-à-vis du transfert d'eau après ultrafiltration d'un fluide dans des conditions opératoires données. Il s'agit donc d'une mesure indirecte des modi- 
fications irréversibles (non éliminées par un lavage à l'eau) subies par la membrane.

\section{Taux de rétention}

Le passage d'un soluté à travers la membrane est caractérisé par le taux de rétention (ou taux de rejet) noté TR et calculé de la façon suivante :

$$
\mathrm{TR}=\left(1-\mathrm{Cp}_{\mathrm{i}} / \mathrm{Cr}_{\mathrm{i}}\right) \times 100
$$

avec $\mathrm{Cp}_{\mathrm{i}}, \mathrm{Cr}_{\mathrm{i}}$ : concentration du constituant i respectivement dans le perméat et dans le rétentat.

Dans le cas d'une rétention idéale, TR $=100$.

Compte tenu de la précision des méthodes analytiques, l'incertitude sur le taux de rétention était : $1,5 \%$ pour le calcium ; $2,5 \%$ pour le lactose et le phosphore, $2 \%$ pour les protéines solubles.

\section{Mode opératoire}

Avant chaque expérience, l'ensemble de l'installation était nettoyé selon la séquence suivante :

- $\mathrm{NaOH} 0,2 \mathrm{M}$ additionnée d'eau de Javel $0,05^{\circ}$ chlorométrique $\left(50^{\circ} \mathrm{C}\right.$, $\left.15 \mathrm{~min}, 2 \mathrm{~m} \cdot \mathrm{s}^{-1}, 2,00.10^{5} \mathrm{~Pa}\right)$;

- rinçage ;

- $\mathrm{HNO}_{3} 0,05 \mathrm{M}\left(50^{\circ} \mathrm{C}, 15 \mathrm{~min}, 2 \mathrm{~m} \cdot \mathrm{s}^{-1}, 2,00.10^{5} \mathrm{~Pa}\right)$;

- rinçage.

Ce cycle de nettoyage était considéré comme efficace si la perméabilité à l'eau de la membrane correspondait à celle trouvée initialement. Au cours de l'expérience d'ultrafiltration, le perméat et le rétentat étaient totalement recyclés dans le bac de recirculation afin que la concentration fût constante (les volumes prélevés pour analyse : 20 à $40 \mathrm{ml}$ au total, étaient négligeables devant le volume total mis en œuvre : 4 à 5 1).

Deux types d'essais étaient conduits :

- une expérience préliminaire permettait de tracer rapidement la courbe $\mathrm{J}=\mathrm{f}(\Delta \mathrm{P})$ à $(\mathrm{V}, \theta)$ donnés sans nettoyage entre chaque point, en incrémentant la valeur de la pression de consigne de $1.10^{5} \mathrm{~Pa}$ toutes les $5 \mathrm{~min}$;

- un suivi du flux d'UF en fonction du temps à $(\mathrm{V}, \Delta \mathrm{P}, \theta)$ donnés. Dans le cas du lactosérum les pressions de consigne étaient choisies d'après le tracé rapide $J=f(\Delta P)$ de telle sorte qu'elles encadraient la pression pour laquelle il y avait apparition d'un flux limite. Pour chaque tracé $J=f(t)$ on suivait le $\mathrm{pH}$ et éventuellement la turbidité du perméat et du rétentat. Des échantillons (5 à $10 \mathrm{ml}$ ) étaient prélevés à $10,45,90$ et $240 \mathrm{~min}$ pour la détermination des taux de rétention.

L'état final de la membrane était caractérisé par une mesure de résistance hydraulique d'après le procotole suivant :

— arrêt de l'expérimentation à un temps donné ;

- vidange de l'installation, rinçage à l'eau distillée pendant $15 \mathrm{~min}$; 
TABLEAU I

Composition (g.l.-1), viscosité dynamique (mPa.s) et $p H$ des solutions de constituants ioniques et non protéiques et du lactosérum doux

Composition (g.l-1), dynamic viscosity (mPa.s) and pH of sweet whey and solutions of ionic and non-proteinic components

\begin{tabular}{|c|c|c|c|c|c|c|c|c|}
\hline \multirow{2}{*}{ Solutions } & \multicolumn{2}{|c|}{$\begin{array}{c}\text { Viscosité } \\
\text { dynamique à }\end{array}$} & \multirow{2}{*}{$\mathrm{pH}$} & \multicolumn{5}{|c|}{ Constituants (g.1-1) (sel utilisé) } \\
\hline & $25^{\circ} \mathrm{C}$ & $50^{\circ} \mathrm{C}$ & & $\begin{array}{c}\mathrm{Ca} \\
\left(\mathrm{CaCl}_{2}\right)\end{array}$ & $\begin{array}{c}\mathrm{P} \\
\left(\mathrm{KH}_{2} \mathrm{PO}_{4}\right)\end{array}$ & $\underset{\left(\mathrm{MgCl}_{2}\right)}{\mathrm{Mg}}$ & $\begin{array}{c}\text { Citrate } \\
\left(\mathrm{Na}_{3} \text { citrate }\right)\end{array}$ & Lactose \\
\hline $\begin{array}{l}A \\
B \\
C \\
D \\
\text { D } \\
F \\
G\end{array}$ & $\begin{array}{l}1,03 \\
1,08 \\
1,11 \\
0,94 \\
0,94 \\
1,04 \\
1,07\end{array}$ & $\begin{array}{l}\bar{z} \\
\bar{z} \\
\bar{z} \\
\overline{0}, 65\end{array}$ & $\begin{array}{l}6,0 \\
6,1 \\
6,9 \\
6,2 \\
6,1 \\
5,9 \\
6,0\end{array}$ & $\begin{array}{l}\overline{0}, 47 \\
0,47 \\
0,47 \\
\overline{-} \\
\overline{0,47}\end{array}$ & $\begin{array}{l}\overline{-} \\
\overline{0,41} \\
\overline{0,41} \\
\overline{0,41}\end{array}$ & $\begin{array}{l}\bar{z} \\
\overline{-} \\
\overline{-} \\
0,12\end{array}$ & $\begin{array}{l}E \\
\bar{E} \\
\bar{E} \\
\overline{1,8}\end{array}$ & $\begin{array}{l}50 \\
50 \\
50 \\
- \\
50 \\
50\end{array}$ \\
\hline Lactosérum & - & 0,66 & 6,3 & $\begin{array}{c}\mathrm{Ca} \\
0,34 \\
\text { matières } \\
\text { azotées } \\
\text { totales } \\
8,11\end{array}$ & $\begin{array}{c}\mathrm{P} \\
0,40 \\
\text { protéines } \\
\text { solubles } \\
\text { totales } \\
6,35\end{array}$ & $\begin{array}{c}\mathrm{Mg} \\
0,11 \\
\begin{array}{c}\beta \text {-lacto } \\
\text { globuline }\end{array} \\
3,84\end{array}$ & $\begin{array}{c}\text { Citrate } \\
1,66 \\
\text { a-lactal } \\
\text { bumine } \\
0,87\end{array}$ & $\begin{array}{c}\text { Lactose } \\
48,3 \\
\text { matières } \\
\text { grasses } \\
\text { * }\end{array}$ \\
\hline
\end{tabular}

* Quantités inférieures à la limite de détection de la méthode $\left(0,005\right.$ g.1 1 l1 $^{-1}$. 
- mesure du flux à l'eau $\left(1,8 \mathrm{~m} \cdot \mathrm{s}^{-1}, 1,00.10^{5} \mathrm{~Pa} ; 25\right.$ ou $50^{\circ} \mathrm{C}$ selon la température de l'essai).

\section{Fluides étudiés}

\section{Solutions modèles}

La concentration des solutions modèles était choisie à partir de la composition moyenne d'un lactosérum doux d'après Alais (1975) et Fevrier (1977) (tabl. 1). Il s'agissait de solutions d'ions $\left(\mathrm{Ca}^{2+}, \mathrm{PO}_{4}{ }^{3-}, \mathrm{Mg}^{2+}\right.$, citrates) avec ou sans lactose. Les réactifs utilisés étaient de qualité «pour analyse»(MERCK).

\section{Lactosérum doux}

Un lot de poudre de lactosérum doux de fromagerie de pâte molle était utilisé afin de disposer du même produit durant toute l'expérimentation (composition donnée en tab1. 1). Le lactosérum était reconstitué à $63 \mathrm{~g}$ d'extrait sec par $\mathrm{kg}$ d'eau distillée et additionné de $\mathrm{NaN}_{3}$ à $1 \%$ pour éviter toute prolifération bactérienne. La reconstitution était effectuée $3 \mathrm{~h}$ avant l'essai. Nous nous sommes assurés qu'il n'y avait pas de dénaturation thermique des protéines solubles au cours des essais d'ultrafiltration.

\section{E. Caractérisation physique et chimique des solutions}

Les concentrations en $\beta$-lactoglobuline et en $\alpha$-lactalbumine étaient déterminées par HPLC (FAUQUANT et al., 1985) (précision $\pm 2,0 \%$ ). Le lactose était dosé par méthode colorimétrique d'après AcToN (1977) (précision $\pm 2,5 \%$ ). La concentration en calcium était déterminée par absorption atomique en présence de chlorure de lanthane selon Brulé et al. (1974) (précision $\pm 0,9 \%$ ). Le phosphore était dosé par colorimétrie (AFNOR, 1980 ; précision $\pm 2,0 \%$ ). La teneur en matières azotées totales (MAT) était déterminée par un dosage de l'azote par microkjeldahl et en multipliant le résultat par 6,38 (OGG, 1960). La teneur en protéines solubles totales était obtenue par différence entre les teneurs en azote non caséinique (NCN) et en azote non protéique (NPN) selon ASCHAFFENBURG et DREWRY (1959). Les matières grasses étaient dosées par la méthode acidobutyrométrique (AMARIGLIO et al., 1973). Enfin, le citrate est dosé par méthode colorimétrique (Pierre et Brulé, 1983).

Le $\mathrm{pH}$ des diverses solutions ou échantillons était mesuré par un pHmètre (précision $\pm 1 \%$, température $20^{\circ} \mathrm{C}$ ).

La viscosité dynamique $(\eta)$ était déterminée par mesure de la viscosité cinématique $(v)$ grâce à un viscosimètre capillaire et par la mesure de la masse volumique à l'aide d'un densimètre. Le produit de ces deux mesures permet de connaître $\eta$ avec une précision de $0,5 \%$.

Les mesures de densité optique à $276 \mathrm{~nm}$ (longueur d'onde déterminée d'après un spectre préalable) effectuées par rapport à un témoin (la solution fraîchement préparée) permettaient d'évaluer l'augmentation relative de la turbidité. 


\section{Résultats}

\section{A. Solutions modèles}

Lors du tracé rapide $\mathrm{J}=\mathrm{f}(\Delta \mathrm{P})$ pour deux vitesses tangentielles $(1,8$ et $3,6 \mathrm{~m} \cdot \mathrm{s}^{-1}$ ) quelles que fussent la solution modèle étudiée et la température ( $25 \mathrm{ou}$ $50^{\circ} \mathrm{C}$ ) on notait que :

- la vitesse de circulation du fluide n'avait pas d'influence sur la valeur du flux d'ultrafiltration;

- pour la gamme explorée de $\Delta \mathrm{P}$, le tracé $\mathrm{J}=\mathrm{f}(\Delta \mathrm{P})$ pouvait toujours être représenté par une droite $(\mathrm{r}=0,999)$;

- la valeur de la pente de ces droites, c'est-à-dire la perméabilité de la membrane vis-à-vis de ces solutions, était toujours plus faible (de 2 à $16 \%$ ) que la perméabilité à l'eau initiale.

La figure 1 montre l'évolution des flux en fonction du temps pour quatre solutions modèles. Aucune rétention de calcium, phosphore ni lactose n'était mise en évidence

La résistance hydraulique finale $\left(\mathrm{Rh}_{\mathrm{f}}\right)$ n'était pas modifiée par le passage des solutions contenant du phosphore et du calcium. Pour les solutions contenant du lactose, on enregistrait par contre des augmentations de $\mathrm{Rh}_{\mathrm{f}}$ de 10 à $20 \%$.

Enfin, dans le cas de la solution $\mathrm{C}$ (lactose $+\mathrm{CaCl}_{2}+\mathrm{KH}_{2} \mathrm{PO}_{4}$ ), on constatait au bout de $8 \mathrm{~h}$ une faible diminution du $\mathrm{pH}$ (de 5,8 à 5,6$)$ et une augmentation de la turbidité qui peuvent s'interpréter par l'apparition d'un précipité de phosphate de calcium. La valeur de $\mathrm{pH}$ de 5,6 correspondait en effet à la limite de solubilité du phosphate de calcium pour les concentrations considérées (LYSTER, 1979). Simultanément, on notait une réduction très sensible de la densité du flux $\left(7,9\right.$ contre $1331 \cdot \mathrm{h}^{-1} \cdot \mathrm{m}^{-2}$ au bout de $\left.2 \mathrm{~min}\right)$, une rétention de calcium et de lactose en fin d'expérience (respectivement TR $=7 \%$ et $13 \%$ ) et une augmentation de la résistance hydraulique finale de la membrane $\left(52,1.10^{12} \mathrm{~m}^{-1}\right.$ contre $7,8.10^{12} \mathrm{~m}^{-1}$ pour la membrane propre).

Après rinçage de la membrane à l'eau distillée, des observations en microscopie électronique ont mis en évidence un dépôt sur la membrane. Des analyses par spectrométrie de photo-électrons émis par rayons $\mathrm{X}$ ont révélé la présence de $\mathrm{P}, \mathrm{O}, \mathrm{Ca}$ et $\mathrm{C}$ que l'on peut attribuer à la formation d'un précipité de phosphate de calcium dans lequel seraient incluses des molécules de lactose à l'origine de l'élément $C$ détecté. Le dépôt formé recouvrait totalement la membrane puisque l'élément $\mathrm{Zr}$ n'était plus détecté.

\section{B. Lactosérum doux}

\section{Densité de flux $(J)$ en fonction de la différence de pression $(\Delta P)$ à vitesse tangentielle donnée}

La courbe $J=f(\Delta P)$ obtenue lors d'un tracé rapide (sans nettoyage avant chaque augmentation de pression) passait par un maximum (fig. 2). Par contre, si l'on reportait (fig. 2) en fonction de la pression les valeurs des densités de flux obtenues à partir des différents tracés $\mathrm{J}=\mathrm{f}(\mathrm{t})$ effectuées pour différents 


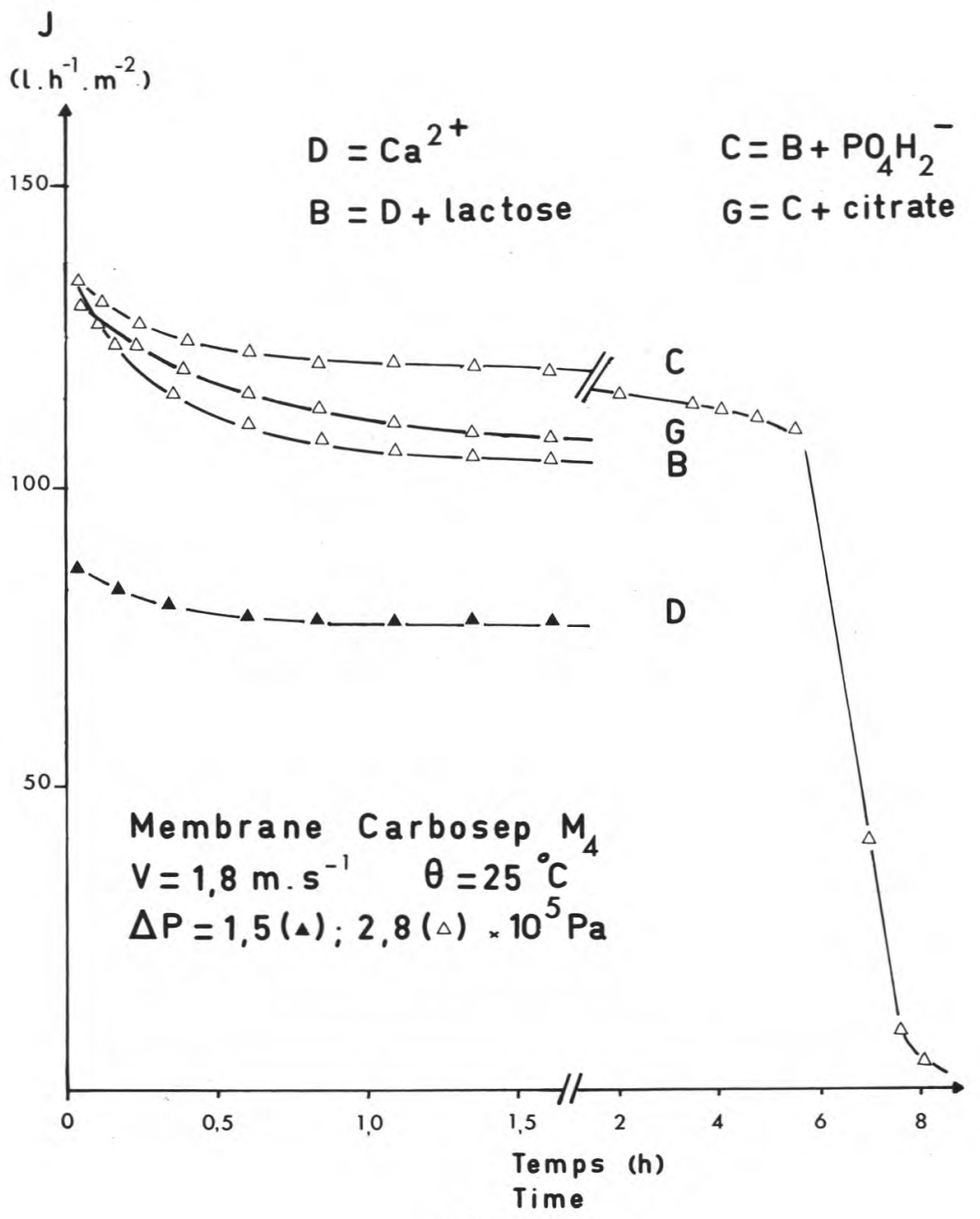

Fig. 1

Evolution des densités de flux $(J)$ en fonction du temps lors de l'ultrafiltration de solutions modèles de solutés ioniques et non protéiques.

Flux density $(J)$ versus time during ultrafiltration of ionic and non proteinic solutions.

couples $(\mathrm{V}, \Delta \mathrm{P})$ et pour des durées variant de $2 \mathrm{~min}$ à $4 \mathrm{~h}$, on notait que les courbes ne passaient plus par un maximum. En outre, l'écart entre les courbes ainsi obtenues diminuait quand le temps augmentait.

2. Influence du temps sur le transfert de matière

L'étude de l'ultrafiltration de lactosérum doux en fonction du temps révélait que, quelles que soient les conditions de fonctionnement choisies, la densité de 


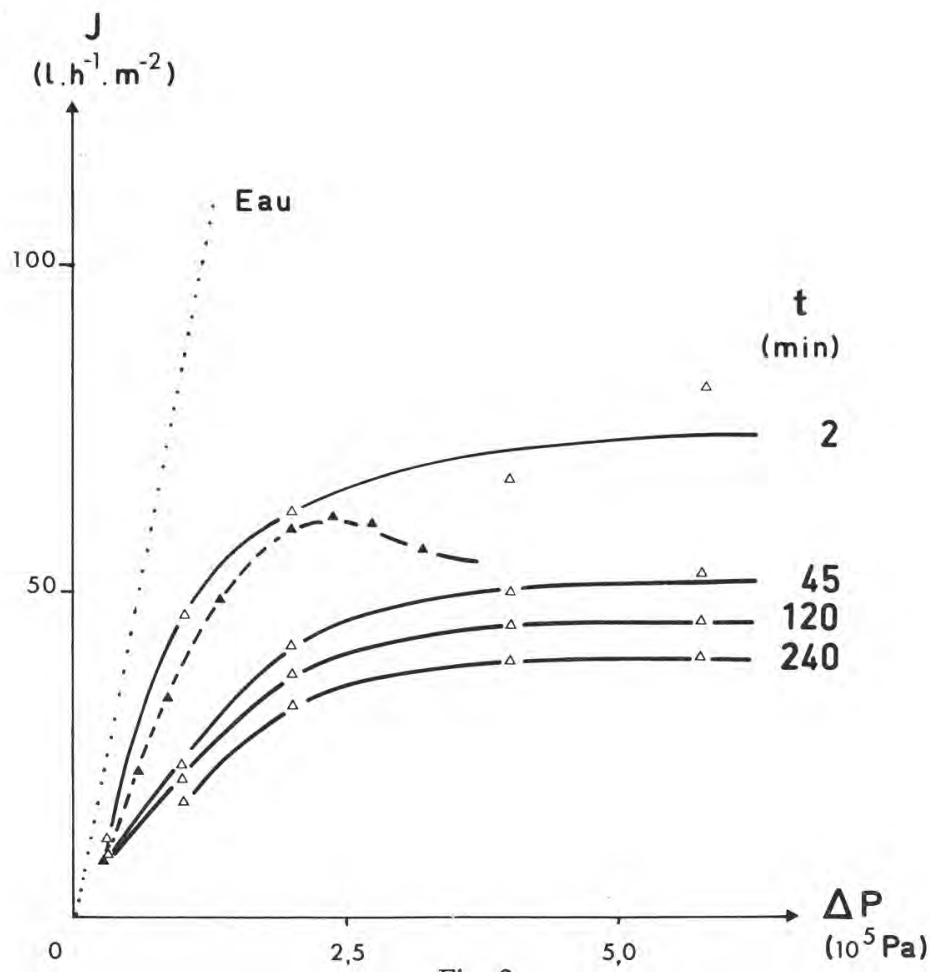

Fig. 2

Caractéristiques $J$ (densité de flux) $=f(\Delta P$ : pression transmembranaire) d'une membrane Carbosep $M_{4}$ (vitesse tangentielle : $1,8 \mathrm{~m} \cdot \mathrm{s}^{-1}$; température : $50^{\circ} \mathrm{C}$ ) :

eau :...

Lactosérum

pH 6,2

6 g. $l^{-1}$

protéines

A: flux mesuré toutes les 5 min sans nettoyage intermédiaire.

$\triangle:$ flux mesuré après diverses durées d'ultrafiltration à $(V, \Delta P$ donnés).

Characteristic $J$ (flux density) $=f(\Delta P$ : transmembrane pressure $)$ for a $M_{4}$ Carbosep membrane (flow velocity : $1.8 \mathrm{~m} . \mathrm{s}^{-1}$; temperature : $50^{\circ} \mathrm{C}$ ) :

water : ...

Sweet whey

pH 6.2

proteins :

6 g.l-1

A: flux measured each five minutes, without intermediate rinsing.

$\triangle:$ flux measured after various ultrafiltration times (on the basis of graph $J=f(t)$ for given $(V, \omega \mathrm{P})$.

flux $(\mathrm{J})$ décroissait toujours rapidement pendant la première heure environ, puis diminuait plus lentement ensuite. Parallèlement, la rétention des protéines variait en fonction des conditions opératoires et de la durée. La rétention de la $\beta$-lactoglobuline (poids moléculaire : 36000 ) était toujours supérieure à celle de l' $\alpha$-lactalbumine (poids moléculaire : 14 000) (fig. 3). Par contre, la rétention du calcium et du lactose était toujours nulle. Enfin, la résistance hydraulique finale était d'autant plus forte que la durée d'ultrafiltration était longue. Au début de la phase initiale de décroissance rapide des flux, les TR des deux protéines analysées 

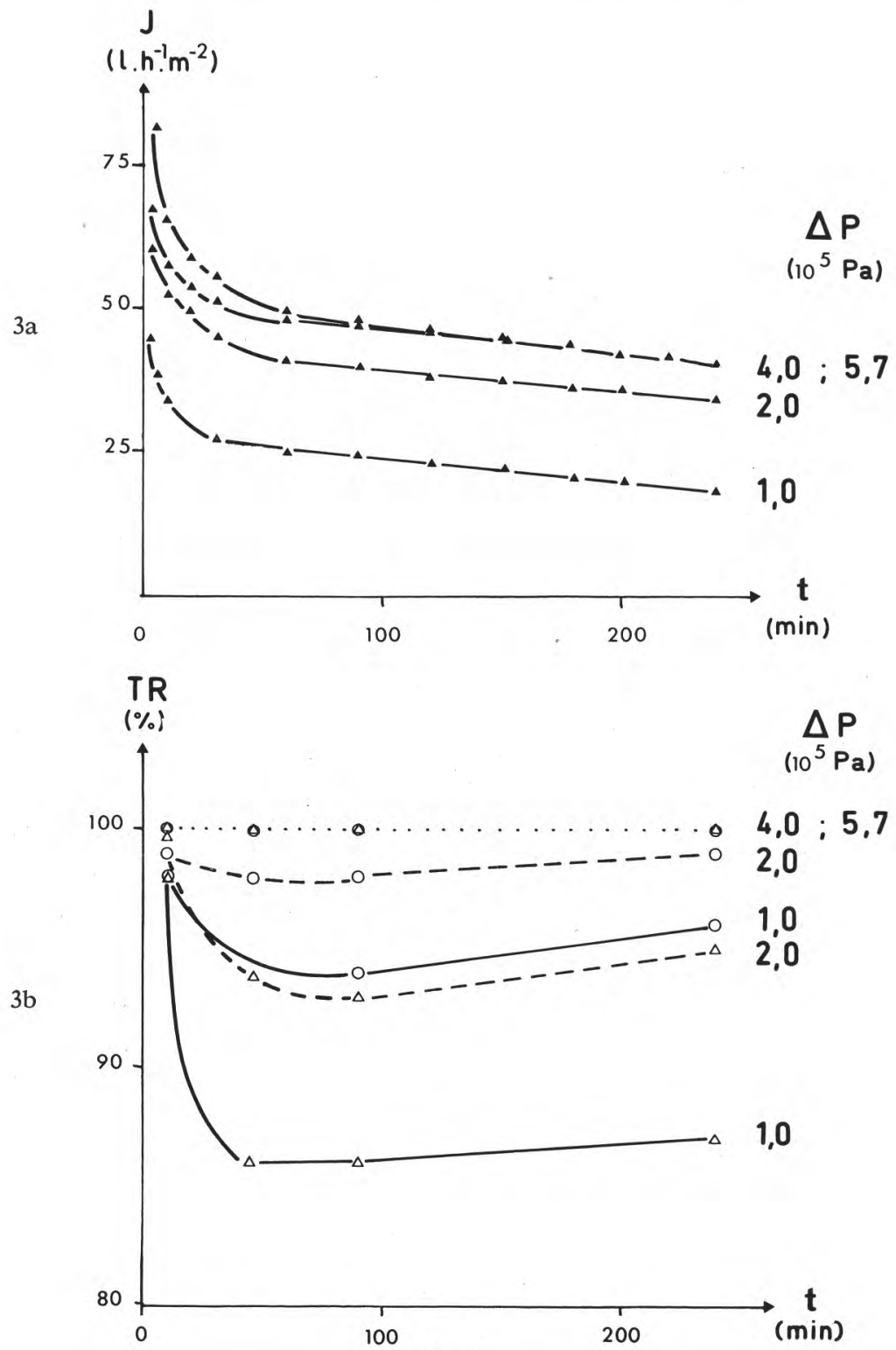

Fig. 3

Effet du temps sur le transfert de matière en ultrafiltration de lactosérum doux (vitesse tangentielle : $1,8 \mathrm{~m} . \mathrm{s}^{-1}$; température : $50^{\circ} \mathrm{C}$ ) sur membrane $M_{4}$ pour diverses pressions transmembranaires.

$3 a-$ Densité de flux $(J)$.

$3 b-$ Taux de rétention $(T R)$ en $\beta$-lactoglobuline $(0)$ et en $\alpha$-lactalbumine $(\triangle)$.

Mass transfer characteristics versus time during sweet whey ultrafiltration (flow velocity: $1,8 \mathrm{~m} \cdot \mathrm{s}^{-1}$; temperature : $50^{\circ} \mathrm{C}$ ) at various transmembrane pressures with $M_{4}$ Carbosep membrane.

$3 a-$ Flux density $(J)$.

$3 b-$ Retention $(T R)$ of $\beta$-lactoglobulin ( $(0)$ and $\alpha$-lactalbumin $(\triangle)$. 


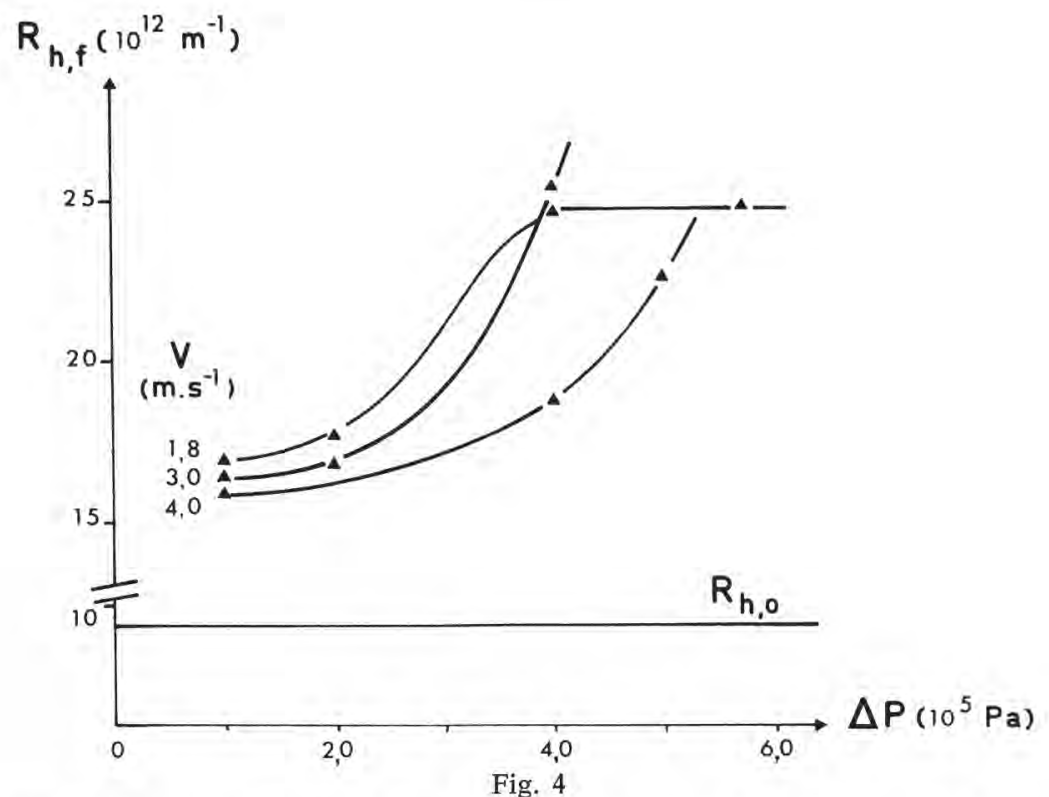

Résistances hydrauliques initiales $\left(R h_{0}\right)$ et finales $\left(R h_{f}\right)$ de la membrane Carbosep $M_{4}$ pour divers couples pression transmembranaire $(\Delta P)-$ vitesse tangentielle $(V)$ utilisés pour l'ultrafiltration de lactosérum doux $\left(4 \mathrm{~h} ; 50^{\circ} \mathrm{C}\right)$.

Initial $\left(R h_{0}\right)$ and final $\left(R h_{f}\right)$ hydraulic resistances of $M_{4}$ Carbosep membrane for various transmembrane pressure $(\Delta P)$ - flow velocity $(V)$ applied during sweet whey ultrafiltration $\left(4 \mathrm{~h} ; 50^{\circ} \mathrm{C}\right)$.

étaient très élevés (97 à $100 \%$ ). Le fait que la concentration en calcium et lactose soit identique dans le perméat et dans le rétentat permettait de vérifier que ce fort taux de rejet n'était pas dû à une dilution des échantillons prélevés à cette durée (10 min). Puis les TR diminuaient, la valeur la plus faible atteinte dans nos essais étant $86 \%$ pour l' $\alpha$-lactalbumine $\left(\Lambda \mathrm{P}=1,00 \cdot 10^{5} \mathrm{~Pa} ; \mathrm{V}=1,8\right.$ ou $\left.3,0 \mathrm{~m} \cdot \mathrm{s}^{-1}\right)$. Au-delà de $60 \mathrm{~min}$, ils augmentaient progressivement sans atteindre systématiquement $100 \%$. La décroissance des flux ralentissait pour devenir linéairement dépendante du temps $(r=0,99)$. Pour l'ensemble des conditions opératoires étudiées, la pente de ces droites pouvait être considérée comme constante et sensiblement égale à $1,67 \cdot 10^{-10} \mathrm{~m} \cdot \mathrm{s}^{-2}$. Les résistances hydrauliques finales $(\mathrm{Rh} \cdot \mathrm{r})$ mesurées après $4 \mathrm{~h}$ d'ultrafiltration étaient 2 à 3 fois supérieures à la valeur de la résistance hydraulique initiale $\mathrm{Rh}$. (fig. 4). En outre, pour une vitesse tangentielle donnée, Rhr était d'autant plus forte que $\Delta P$ utilisé pour l'ultrafiltration était élevé (fig. 4), montrant ainsi une aggravation du colmatage de la membrane sous l'effet de la pression. Cependant, pour la vitesse la plus faible utilisée $\left(1,8 \mathrm{~m} \cdot \mathrm{s}^{-1}\right), \mathrm{Rh}$ !n'augmentait plus quand $\Delta \mathrm{P}$ passait de 4,00 à $5,70.10^{5} \mathrm{~Pa}$. Par ailleurs, à pression donnée, $\mathrm{Rh}_{\mathrm{f}}$ était plus élevée pour les faibles valeurs de la vitesse tangentielle.

Sur la figure 5 , on constate que la résistance hydraulique finale augmentai brutalement lorsque les valeurs de densité de flux se rapprochaient de la valeuı 


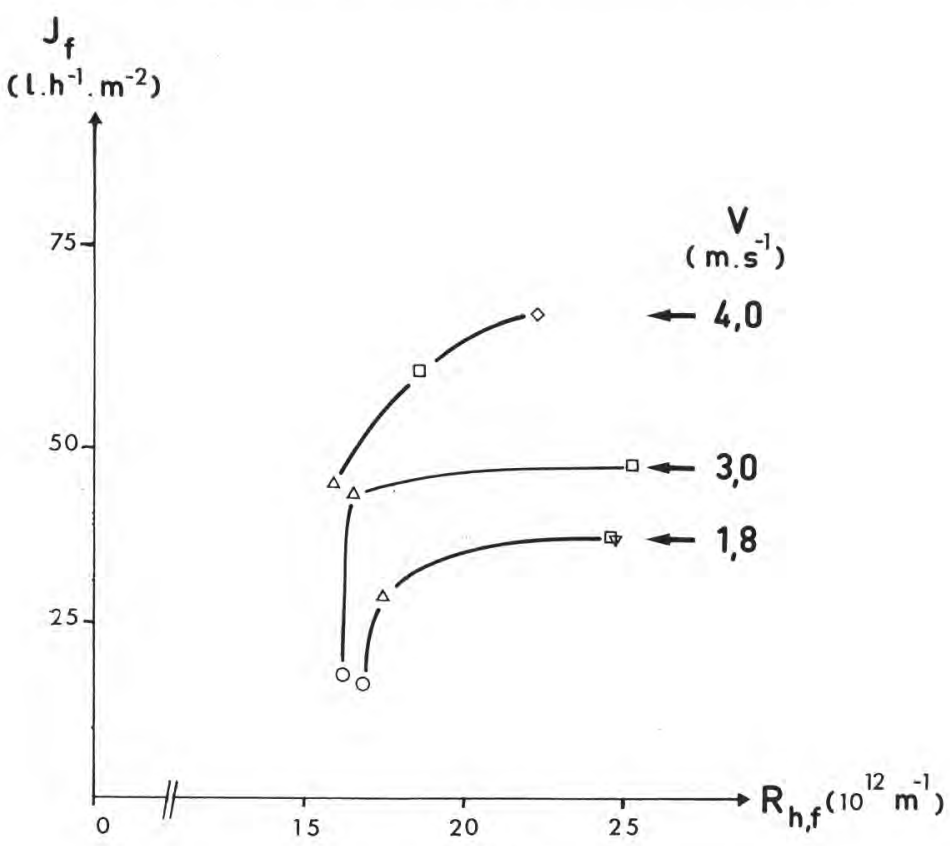

Fig. 5

Densité de flux final $(J f)$ en fonction de la résistance hydraulique finale $\left(R h_{f}\right)$ relevée après $4 \mathrm{~h}$ d'ultrafiltration de lactosérum doux $\left(50^{\circ} \mathrm{C}\right)$ sur membrane Carbosep $M_{4}$ réalisées pour 3 vitesses tangentielles $(V)$ et les différences de pression transmembranaires suivantes : $100(0) ; 2,00(\triangle) ; 4,00(\square) ; 5,00(\diamond) ; 5,70(\nabla) 105 \mathrm{~Pa}$.

Final flux density $(J f)$ versus final hydraulic resistance $\left(R h_{f}\right)$ measured after 4 hours sweet whey ultrafiltration with $M_{4}$ Carbosep membrane at 3 tangential flow velocities $(V)$ and following transmembrane pressures : $1.00(0) ; 2.00(\triangle) ; 4.00(\square) ; 5.00(\diamond)$; $5.70(\nabla) 105 P a$.

limite (voir fig. 2). Une valeur limite de la résistance hydraulique est également atteinte : $25 \cdot 10^{12} \mathrm{~m}^{-1}$ à $1,8 \mathrm{~m} \cdot \mathrm{s}^{-1}$ (fig. 5).

Enfin, la figure 6 fait apparaître une corrélation entre les taux de rétention pour les deux protéines considérées et la résistance hydraulique mesurée dans les mêmes conditions. Il est intéressant de noter qu'au-delà d'une valeur seuil de $\mathrm{Rh}_{\mathrm{r}}$ les taux de rétention pour la $\beta$-lactoglobuline et l' $\alpha$-lactalbumine sont de $100 \%$; en deçà de cette valeur, ils diminuent et deviennent de plus en plus différents l'un de l'autre.

\section{Discussion}

A. Caractérisation de l'état initial et final de la membrane

Suivant l'origine et la nature de l'eau utilisée pour effectuer des mesures de résistance hydraulique, des diminutions non négligeables de la perméabilité de 


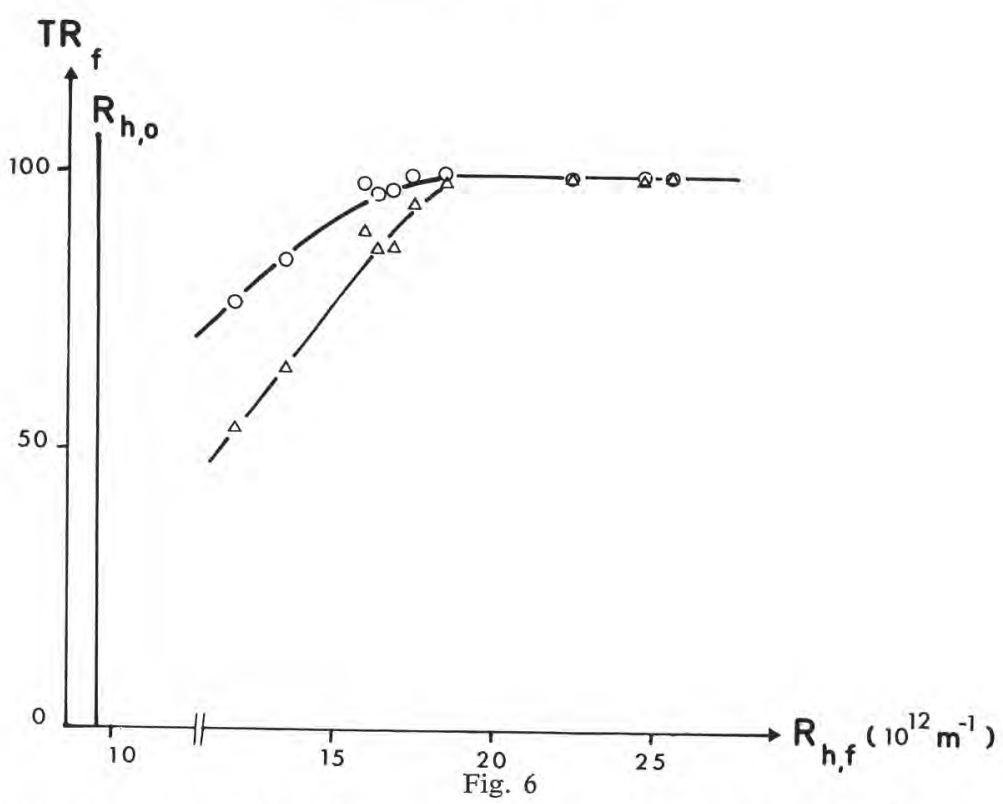

Taux de rétention final $(T R f)$ de $\beta$-lactoglobuline $(0)$ et $\alpha$-lactalbumine $(\Delta)$ en fonction de la résistance hydraulique finale $\left(R h_{f}\right)$ relevés après $4 \mathrm{~h}$ d'ultrafiltration de lactosérum doux $\left(50^{\circ} \mathrm{C}\right)$ sur membrane Carbosep $M_{4}$.

Final retention $(T R f)$ of $\beta$-lactoglobuline $(O)$ an $\alpha$-lactabumine $(\triangle)$ versus final hydraulic resistance $\left(R h_{f}\right)$ after $4 \mathrm{~h}$ sweet whey ultrafiltration $\left(50^{\circ} \mathrm{C}\right)$ with $\mathrm{M}_{4}$ Carbosep membrane.

la membrane propre étaient observées au cours du temps. Ces résultats sont conformes aux observations réalisées sur membranes polymères par FANE (1983) et sur membranes de microfiltration en alumine par ZiDoune (1983).

Vraisemblablement, la présence dans l'eau de micro-organismes ou de colloïdes (FANE, 1983) joue un rôle non négligeable sur le transfert d'eau.

Il est à noter qu'un temps de 15 min est nécessaire pour atteindre une valeur stable de la résistance (la variation de $\mathrm{Rh}$ entre l'instant zéro et l'instant $15 \mathrm{~min}$ est de quelques pour-cent). Ce laps de temps est le même pour celui déterminé par BAKLOUTI et al. (1984) dans le cas de l'adsorption d'albumine de sérum bovin sur membranes organiques.

\section{B. Ultrafiltration de solutions modèles}

L'ultrafiltration de solutions modèles révélait que la perméabilité de la membrane vis-à-vis de ces solutions était plus faible que la perméabilité à l'eau. Les mesures de viscosité de ces solutions effectuées à $25^{\circ} \mathrm{C}$ ou à $50^{\circ} \mathrm{C}$ (tabl. 1) montrent que la relation suivante est vérifiée [tirée des équations (1) et (2)] :

$$
\frac{\text { Lp eau }}{\text { Lp solution }}=\frac{\eta \text { solution }}{\eta \text { eau }} \text { (coefficient de corrélation : 0,99) }
$$


Ceci montre que la membrane Carbosep se comporte comme un milieu poreux filtrant sans sélectivité particulière vis-à-vis de solutions contenant les solutés non protéiques du lactosérum. Duchesnay (1982) avait également mis en évidence l'absence de rétention de solutés non protéiques du lactosérum sur membrane en oxyde de zirconium, exception faite pour certains anions d'acide faible pour des $\mathrm{pH}$ neutres ou alcalins.

Néanmoins la présence de lactose entraînait une diminution des vitesses de perméation au cours du temps de l'ordre de 10 à $20 \%$, conduisant à une légère augmentation de la résistance hydraulique. Ceci pourrait s'expliquer par des phénomènes d'adsorption de ce constituant dans la membrane.

Le cas particulier de la solution $\mathrm{C}$ (lactose $+\mathrm{CaCl}_{2}+\mathrm{KH}_{2} \mathrm{PO}_{4}$ ) ultrafiltrée pendant $7 \mathrm{~h}$ montre qu'une faible variation de $\mathrm{pH}$ accompagnée d'un léger précipité de phosphate de calcium peuvent gravement affecter les performances de la membrane. Ce résultat est intéressant car la précipitation de phosphate de calcium est considérée par MERIN (1979) et LEE (1977) comme l'un des agents responsables du colmatage des membranes lors de l'ultrafiltration de lactosérum. Il ne faut donc pas modifier les équilibres ioniques du lactosérum en cours d'ultrafiltration.

\section{Ultrafiltration de lactosérum}

La décroissance des densités de flux était tout d'abord rapide. Elle ralentissait ensuite pour devenir linéaire. Kuo et CHERYan (1983) ayant observé une allure semblable dans le cas de l'ultrafiltration de lactosérum sur membrane polymère insérée dans un module spirale ont proposé un modèle phénoménologique dans lequel $\mathrm{J}$ est proportionnel à $\mathrm{t}^{-\mathrm{b}}(\mathrm{t}$ : temps et $\mathrm{b}>0$; exposant propre à une membrane pour un fluide et des conditions données). Mais, malgré un excellent accord avec l'expérience, ce modèle ne renseigne pas sur les mécanismes responsables de la décroissance des flux.

Durant cette première période, les taux de rétention en protéines et les densités de flux variaient dans le même sens. Cette évolution peut être considérée comme la conséquence d'un phénomène d'adsorption ainsi que FANE et al. (1983) et Howell et al. (1981) l'ont proposé pour interpréter une évolution semblable dans le cas de l'ultrafiltration de solutions de protéines sur membranes polymères. Cependant, cette hypothèse n'a pas encore trouvé de confirmation expérimentale. En effet, compte tenu de la précision des méthodes analytiques, un calcul du bilan matière sur les protéines ne permet pas de mettre en évidence une adsorption de ces dernières sur la membrane.

Toutefois, il est probable que la formation de la couche de polarisation est très rapide. CHUDACEK et FANE (1984) ont montré que le temps nécessaire à la mise en place de ce phénomène limitant était inférieur à $1 \mathrm{~min}$. Ceci est en accord avec les résultats de la figure 2 qui révèlent qu'au bout de $2 \mathrm{~min}$ la loi de Darcy n'est déjà plus suivie. En outre, Jonsson (1983) et Vilker (1984) ont montré que l'effet de la polarisation dans le cas de l'ultrafiltration de solution de protéines se traduit par une pression osmotique du même ordre de grandeur que la pression appliquée et opposée à celle-ci.

En conséquence, l'adsorption initiale et l'établissement d'une couche de polarisation susceptible d'engendrer une pression osmotique pourraient expliquer les 
variations de flux et de rétention de protéines observées pendant la première heure d'ultrafiltration.

Puis, durant une deuxième période, la baisse des flux ralentit et la rétention des protéines s'améliore après être passée par un minimum (fig. 3). La courbe $J=f(t)$ devient alors linéaire $(1 \mathrm{~h}<\mathrm{t}<4 \mathrm{~h})$. Howell et al. (1981) ont tenté d'expliquer la décroissance plus lente des flux par la formation et la croissance d'un gel. D'après la théorie du gel développée par Blatt et al. (1970), la formation d'un gel n'intervient que pour une concentration à la membrane donnée et atteinte seulement pour une pression telle qu'il y ait apparition d'un flux limite. Or nos travaux montrent que les courbes $\mathrm{J}=\mathrm{f}(\mathrm{t})$ présentaient toujours la même allure qu'elles aient été réalisées ou non dans des conditions de flux limite. II semblerait que pour interpréter l'évolution des flux et des taux de rétention il faille supposer l'existence d'un autre phénomène limitant qui se surimposerait aux précédents : une accumulation de matière sous la forme d'un dépôt superficiel composé probablement d'insolubles phosphocalciques et d'agrégats protéiques pouvant s'accompagner de la formation d'un gel si la concentration en protéines atteinte au voisinage de la membrane, en fonction des conditions opératoires et de la durée, le permet.

Ainsi, l'augmentation de la vitesse tangentielle (à $\Delta \mathrm{P}$ donné) entraîne un accroissement des débits d'ultrafiltration mais diminue la rétention en protéines et la résistance hydraulique (fig. 4). Donc, certains des phénomènes qui contribuent à la rétention des protéines sont sensibles au régime hydrodynamique et par conséquent ont lieu en surface.

Ainsi également, l'augmentation de $\Delta \mathrm{P}$ nécessaire pour améliorer les flux (à $\mathrm{V}$ donnée) entraîne une amélioration de la rétention mais aussi une aggrayation du colmatage puisque la résistance hydraulique finale augmente. Par contre, lorsque la pression n'a plus d'effet sur le flux $\left(1,8 \mathrm{~m} \cdot \mathrm{s}^{-1} ; \Delta \mathrm{P}>4,00.10^{5} \mathrm{~Pa}\right)$, elle ne semble pas en avoir non plus sur la résistance hydraulique finale (fig. 4), ni sur la rétention en protéines. Ces observations ne peuvent s'interpréter par des modifications des caractéristiques de la membrane (adsorption, particules en suspension). En outre, d'après la théorie du gel, au flux limite, un accroissement de $\Delta \mathrm{P}$ s'accompagne d'un accroissement de l'épaisseur du gel donc de la résistance au transfert de solvant. Ceci est en désaccord avec nos résultats expérimentaux; les courbes $J=f(t)$ obtenues pour des pressions supérieures à celle correspondant à un flux limite $\left(4,00\right.$ et $5,70.10^{5} \mathrm{~Pa}$ pour $\left.1,8 \mathrm{~m} . \mathrm{s}^{-1}\right)$ se confondent quasiment au-delà de 1 h 30 (fig. $3 \mathrm{a}$ ) et la résistance hydraulique mesurée après ces expériences n'augmente pas (fig. 4). Par contre, l'existence d'une pression osmotique qui contrebalance la pression appliquée expliquerait ces observations.

Ainsi, enfin, les résultats reportés sur la figure 6 soulignent que les phénomènes qui participent au colmatage (augmentation de $\mathrm{Rh}$ ) contribuent également à la rétention de protéines. On peut donc raisonnablement penser que l'hypothèse que nous avons avancée : accumulation de matière sous la forme d'un dépôt superficiel accrôit la résistance au passage de l'eau et limite le passage des protéines par effet tamis. La figure 6 montre en outre l'existence d'une valeur-seuil de la résistance hydraulique au-delà de laquelle la rétention de la $\beta$-lactoglobuline et de l' $\alpha$-lactalbumine par la membrane est totale. Ceci peut s'expliquer par l'existence d'un dépôt à la structure trop serrée pour laisser passer les protéines sériques, ou par une diminution suffisante du diamètre moyen des pores. Une analyse simultanée des courbes $\mathrm{J}=\mathrm{f}(\mathrm{t})$ et $\mathrm{TR}=\mathrm{f}(\mathrm{t})$, en relation avec les hypo- 
thèses que nous avons formulées, est en cours pour tenter de modéliser les phénomènes.

A partir de nos résultats, nous avons résumé l'influence des paramètres opératoires $(\mathrm{V}, \Delta \mathrm{P}, \mathrm{t})$ sur le transfert de solvant en ultrafiltration $(\mathrm{J})$, le transfert de protéines (TR) et le colmatage $(\mathrm{Rh})$ dans le tableau 2. Nous pouvons en déduire quelques propositions sur la conduite d'une installation d'ultrafiltration.

\section{TABLEAU II}

Evolution de la densité de flux (J), du taux de rétention en protéine (TR) et de la résistance hydraulique $(R h)$ de la membrane en fonction des conditions d'ultrafiltration : pression transmembranaire $(\Delta P)$, vitesse tangentielle $(V)$ et durée $(t)$

Evolution of permeate flux density $(\mathrm{J})$, retention $(T R)$ and hydraulic resistance $(R h)$ of membrane with operating parameters : transmembrane pressure $(\Delta P)$, flux velocity $(V)$ and time $(t)$

\begin{tabular}{|c|c|c|c|}
\hline $\begin{array}{l}\text { Ultrafiltration } \\
\text { de lactosérum doux } \\
\left(50^{\circ} \mathrm{C} \text {, Carbosep } \mathrm{M}_{4}\right)\end{array}$ & $\mathrm{J}$ & $\begin{array}{c}\text { TR } \\
\text { protéines }\end{array}$ & $\mathrm{Rh}$ \\
\hline$\Delta \mathrm{P} \nearrow$ à $\mathrm{V}$ cte & $\pi \rightarrow$ & $\lambda$ & $\lambda \rightarrow$ \\
\hline $\begin{array}{c}\Delta \mathrm{P}<2.10^{5} \mathrm{~Pa} \\
\mathrm{~V} \underset{\mathrm{a}}{\Delta \mathrm{P}} \mathrm{cte} \\
\Delta \mathrm{P}>2.10^{5} \mathrm{~Pa}\end{array}$ & 7 & $\rightarrow$ & $\begin{array}{l}\rightarrow \\
\searrow\end{array}$ \\
\hline t $\nearrow$ à $(V, \Delta P)$ cte & $\searrow$ & $\pi$ & $\lambda$ \\
\hline
\end{tabular}

Une solution, utilisée au plan industriel, consiste à opérer à $\Delta \mathrm{P}$ constante et à choisir un débit de perméat $\mathrm{J}$ inférieur de 20 à $30 \%$ au flux limite. La résistance hydraulique évolue alors lentement avec le temps, ce qui permet d'augmenter la durée du cycle tout en ayant un taux de rétention moyen relativement élevé.

Compte tenu de la baisse du flux $\mathrm{J}$ au cours du temps, une autre solution adoptée plus récemment par les industriels consiste à maintenir le débit de perméat constant et par conséquent à augmenter $\Delta \mathrm{P}$ pour une vitesse tangentielle donnée. Ceci a pour avantage de favoriser la rétention des protéines mais pour inconvénient d'aggraver le colmatage. Il en découle que, si la valeur choisie pour J est élevée, la durée du cycle peut être très courte.

Une troisième possibilité consisterait à agir préférentiellement sur la vitesse pour une différence de pression transmembranaire donnée. Elle conduit à un colmatage limité mais à des taux de rétention plus faibles (sauf si $\Delta \mathrm{P}<2,00.10^{5} \mathrm{~Pa}$, dans ce cas l'augmentation V n'a pas d'effet sur la rétention). En outre, il faudrait effectuer une analyse économique pour déterminer si cette solution est préférable aux solutions actuelles. La manière de conduire une installation d'ultrafiltration dépend toutefois de critères qui doivent être fixés par avance : absence de fuites 
protéiques dans le perméat, concentration rapide de volumes donnés, réduction des cycles de nettoyage, etc. On peut également être amené à rechercher des compromis tels qu'une rétention protéique maximale, ce qui revient à choisir des conditions de fonctionnement $(\mathrm{V}$ et $\Delta \mathrm{P})$ telles que la résistance seuil mise en évidence sur la figure 6 soit atteinte.

Enfin, une analyse plus fine du rôle des paramètres opératoires montre que l'on peut contrôler la rétention dès le début de l'expérience. On peut, par exemple, la maintenir à son niveau maximal en commençant à pression relativement élevée et vitesse lente. L'essai est alors poursuivi en augmentant la vitesse tangentielle, se qui se traduit en pratique par une élévation modérée de la pression due aux pertes de charge, sans pour autant augmenter fortement la résistance hydraulique. On peut alors maintenir le flux et la rétention à un niveau donné.

La résistance hydraulique d'une membrane donnée varie en fonction de la nature du produit à ultrafiltrer et des conditions opératoires. Il est impossible de calculer sa valeur pour un produit donné dans l'état actuel de nos connaissances sur l'ultrafiltration. Cependant, la méthodologie que nous avons mise au point (suivi simultané des flux, de la rétention en protéines et de la résistance hydraulique) permet, à l'aide d'un minimum d'expériences, d'affiner le choix des conditions opératoires suivant les critères recherchés par l'utilisateur. Pour cela, il convient d'effectuer un tracé rapide de $J=f(\Delta P)$ pour fixer l'ordre de grandeur des couples $(\mathrm{V}, \Delta \mathrm{P})$ qui seront choisis et de réaliser des expérimentations pour évaluer l'incidence des conditions opératoires sur le transfert de matière. En effet, les courbes $J=f(\Delta P)$, dépendant fortement du protocole expérimental, ne peuvent être considérées comme caractéristiques d'un système que si les conditions et le protocole opératoires sont clairement définis.

Enfin, il est également nécessaire de rechercher l'influence de la concentration initiale en protéines sur les grandeurs étudiées. Ceci devrait permettre d'affiner les hypothèses concernant les phénomènes responsables des iimitations du transfert de matière et de mieux extrapoler nos résultats pour la conduite d'installations d'ultrafiltration.

\section{Conclusion}

L'étude de l'évolution du transfert de solutés et de solvant au travers d'une membrane minérale en fonction des conditions opératoires (vitesse tangentielle, différence de pression transmembranaire moyenne) et du temps contribue à améliorer les connaissances sur les phénomènes intervenant pendant l'ultrafiltration au plan des mécanismes. L'évolution de la résistance hydraulique de la membrane inhérente à un essai d'ultrafiltration permet de suivre le colmatage et le rôle que joue ce paramètre sur les taux de rétention des protéines et les densités de flux de solvant.

Lors de l'ultrafiltration de solutions modèles ou du lactosérum doux à une concentration d'environ 6 g. . $^{-1}$ en protéines, les petits solutés $(\mathrm{PM}<1000)$ du lactosérum ne sont pas retenus par la membrane utilisée. Le rôle de la viscosité nettement mis en évidence dans le cas des solutions modèles est masqué par d'autres phénomènes limitant le transfert de matière pour du lactosérum à $6 \mathrm{~g} / \mathrm{l}^{-1}$ en protéines. Toutefois, l'apparition de matières en suspension (précipité minéral 
de phosphate de calcium), par suite d'un déplacement de l'équilibre ionique, entraîne une chute de débit même pour des quantités très faibles de précipité.

Au cours des premières minutes de l'ultrafiltration du lactosérum doux, des phénomènes d'adsorption et la mise en place d'une couche de polarisation expliqueraient la réduction rapide des flux et la diminution généralement observée des taux de rétention. Ensuite, pour des durées plus longues, l'accumulation de matière sur et dans la membrane rendrait compte de l'augmentation de la rétention en protéines et de la diminution plus lente des flux. Une augmentation de la pression transmembranaire pour une pression inférieure à la pression d'apparition du flux limite ou une diminution de vitesse entraîne une élévation parfois très forte de la résistance hydraulique (surtout si le flux tend vers le flux limite) et du taux de rétention. Dès lors l'accumulation de matière sur la membrane devient importante et participe à la filtration de macromolécules. Par contre, une augmentation de la vitesse tangentielle peut être sans effet sur les taux de rétention pour une pression faible; elle diminue simplement l'épaisseur de la couche de polarisation et provoque une augmentation de la vitesse de filtration. Le taux de rétention est donc une grandeur qui, si elle dépend des propriétés de la membrane, varie également avec les conditions opératoires et le temps. Ainsi, les résultats de cette étude ont permis de mieux cerner les phénomènes limitant le transfert.

Enfin, l'évaluation de l'évolution simultanée de la densité de flux, du taux de rétention et de la résistance hydraulique en fonction des conditions opératoires constitue une méthodologie facilement utilisable pour caractériser les performances de tout autre couple membrane-solution.

\section{Remerciements}

Nous tenons à remercier nos collègues du Laboratoire de Recherches de Technologie laitière (I.N.R.A. Rennes) : J.-L MAUBOIS et G. BRULE pour leurs conseils scientifiques et Y. LE GRAET et Mme Françoise MICHEL pour leur collaboration technique.

Nous remercions également A. QUEMERAIS (Laboratoire de Spectroscopie, Université Rennes I) pour les analyses en spectroscopie de photo-électrons X et C. DASTE (Ecole nationale supérieure de l'Aéronautique et de l'Espace, Toulouse) pour les observations effectuées en microscopie électronique.

Reçu le 21 mars 1986.

Accepté pour publication le 16 juin 1986.

\section{Références bibliographiques}

Acton (G.H.), 1977. Determination of lactose in cheese. Aust. J. Dairy Technol., 32, 111. AFNOR, 1980. Dosage du phosphore. NF V 04-284, septembre 1980.

Alais C., 1975.. In : Science du lait. Principes des techniques laitières. Ed. Publicité.

Amariglio S., Serre S.L., Petranxienne D., 1973. Contrôle de la qualité des produits laitiers. I. Analyse physique et chimique. Direction des services vétérinaires. Laboratoire Central de l'Inspection du lait. 
Amundson C.H., Watanawanichakorn S., Hill C.G., 1982. Production of enriched protein fractions of $\beta$-lactoglobuline and $\alpha$-lactalbumine from cheese whey. J. Food Process. Preserv., 6, 55-71.

AschafFEnBURG R., DREWRY J., 1959. New procedure for the routine determinations of the various non casein proteins of milk. Int. Dairy Cong., London, 3, 1631-1637.

Baklouti S., AtMaR P., SANChez V., 1984. Adsorption et transfert de matière en ultrafiltration. Lait, 64, 206-216.

BELfORT G., MARX B., 1979. Artificial particulate fouling of hyperfiltration membranes. Desalination, 28 (1), 13-30.

Blatt W.F., Dravid A., Michaels A.S., Nelson L., 1970. In : Membrane Science and Technology. J.E. Flinn. Ed. Plenum Press, New York, 47-97.

Brulé G., Maubors J.L., Fauquant J., 1974. Etude de la teneur en éléments minéraux des produits obtenus lors de l'ultrafiltration de lait sur membrane. Lait, 54, 600-615.

CHUDACEK M.W., FANE A.G., 1984. The dynamics of polarisation in unstirred and stirred ultrafiltration. J. Membrane Sci., 21, 145-160.

Clifton M.J., ABIDINE N., APTEL P., SANCHEZ V., 1984. Growth of the polarization layer in ultrafiltration with hollow fiber membranes. I. Membrane Sci., 21, 233-246.

DUCHESNAY B., 1982. Rôle des différents constituants du lactosérum sur la perméabilité et la sélectivité d'une membrane d'ultrafiltration. Mém. fin Et, Univ. Haute-Bretagne, Brest (France).

FANE A.G., 1983. Factors affecting flux and rejection in ultrafiltration. J. Separ. Proc. Technol., 4 (1), 15-23.

FANE A.G., Fell C.D.J., WATERS A.G., 1983. Ultrafiltration of proteins solutions through partially permeable membranes. The effect of adsorption and solution environment. J. Membrane Sci., 16, 211-224.

FANE A.G., 1984. Ultrafiltration of suspensions. J. Membrane Sci., 20, 249-259.

Fauguant J., Vieco S., Brulé G., Maubois J.L., 1985. Clarification des lactosérums doux par agrégation thermocalcique de la matière grasse résiduelle. Lait, 65 (647-648), 1-20.

FÉVRIER, 1977. Lactosérum et sous-produits laitiers dans l'alimentation du porc. Ed. Inst. Techn. Porc, Série I.

GOURDON P., 1980. Effet des caractéristiques physico-chimiques des solutions à filtrer sur les performances du matériel d'ultrafiltration. Mém. fin Et., E.N.S.A. Rennes (France).

HICKEY M.W., HILL R.D., SмITH B.R., 1980. Investigation into the ultrafiltration and osmosis of whey : the effects of certain pretreatments. N. Z. Dairy Technol., 15 (2), 109-121.

Howell J.A., Velicangil O., Le M.S., Herrera A.L., 1981. Uitrafiltration of protein solutions. Ann. New York Acad. Sci., 369, 355-366.

Jonsson G., 1983. Boundary layer phenomena during ultrafiltration of dextran and whey proteins solutions. Fourth symp. on synthetic membranes in science and industry, Tubingen, sept. 6-9.

KUo K.P., CHERYAN M., 1983. Ultrafiltration of acid whey in a spiral wound unit. Effect of operating parameters on membrane fouling. I .Food Sci., 48 (4), 1113-1117.

LAFAILLE J.P., 1984. Concentration et purification de macromolécules par ultrafiltration. Thèse d'Université $\mathrm{n}^{\circ}$ 368. Université Paul-Sabatier, Toulouse (France).

LEE R.C., 1977. Serum factors affecting permeation and fouling of selected UF membranes. $\mathrm{Ph}$. D. Thesis, Ohio state University, Ohio (U.S.A.).

LYSTER R.L.J., 1979. The equilibrium of calcium and phosphate ion with the micellar calcium phosphate in cow's. J. Dairy Res., 46, 343-346.

MATTHIASSON E., 1983. The role of macromolecular adsorption in fouling of ultrafiltration membranes. J. Membrane Sci., 16, 23-36.

MauboIs J.L., 1982. Les protéines du lactosérum extraites par ultrafiltration In : Protéines animales, extraits, concentrés et isolats en alimentation humaine. Bourgeois C.T. et Le Roux P., éd., Aria-Lavoisier, Paris. 172-190.

Merin U., 1979. A study of the mechanism of fouling of ultrafiltration membranes. Ph. D. Thesis, Univesity of Illinois at Urbana Champaign, Illinois (U.S.A..).

OGG C.L., 1960. Determination of nitrogen by the micro-kjeldahl. J. Assoc. Off. Agric. Chem., 43, 689-693.

Pierre A., Brulé G., 1983. Dosage rapide du citrate dans l'ultrafiltrat de lait par complexation cuivrique. Lait, 63, 66-74.

VILKER V.L.., 1984. The osmotic pressure of concentration protein and lipoprotein solutions and its significante to ultrafiltration. J. Membrane Sci., 20, 63-77.

ZIDOUNE M.N., 1983. Etude de l'ultrafiltration des lactosérums sur membranes minérales. Thèse Docteur-Ingénieur, U.S.T.L. Montpellier (France). 